\title{
Effects of boundary layer displacement and separation on opposed-flow flame spread
}

\author{
F J Higuera, A Liñán and I Iglesias†
}

\begin{abstract}
An analysis is presented of the viscous-inviscid interaction region around the tip of a flame spreading over the surface of a solid fuel in a forced laminar high Reynolds number air stream that opposes the flame propagation. Through the interaction, the vaporization of the solid and the thermal expansion of the gas originate an adverse pressure gradient upstream of the flame tip, which leads to a decrease of the shear acting on the small region controlling the flame spread rate. Under certain realistic conditions this adverse pressure gradient may separate the boundary layer upstream of the vaporizing region of the solid, leading to a new mode of flame spread with a higher spread rate determined by the flow in the whole interaction region.
\end{abstract}

\section{Introduction and orders of magnitude}

The problem of flame spread over a solid fuel in a forced stream of oxidizing gas opposing the flame propagation has been much studied in the past. In their pioneering work, Tarifa and Torralbo [1] computed the flame spread rate assuming that the combustion in the gas creates a known distribution of heat flux on the solid surface and solving the heat conduction problem in the solid. Taking into account heat conduction in both phases and solving the gas-phase problem with the Oseen approximation, deRis [2] obtained flame spread rate formulae for thermally thin and thermally thick solids based on the assumption of diffusion controlled combustion. This author also realized the existence of a quenched distance between the flame and the solid surface and discussed the triple-flame problem for flame spread for the first time. The work of deRis has been very much extended and refined subsequently; see references $[3,4,5,6]$ for reviews of the existing theoretical and experimental results. The problem will be further analysed here in the framework of the interacting boundary layer theory, assuming that the forced gas flow is laminar at high Reynolds number and that the gasification of the fuel is an endothermic surface process occurring at a constant vaporization temperature. In addition, body forces and radiation will be neglected and the solid will be assumed to be thermally thick.

What follows is a summary of well known order of magnitude estimations, with extensions to the problems tackled in the paper; further details can be found in [7]. The subscripts $s$ and $g$ denote magnitudes in the solid and gas phases, respectively (far upstream of the flame when these magnitudes depend on the local temperature), $U_{\infty}$ is the free stream velocity, and $T_{\infty}$ is the ambient temperature. A reference frame moving with the velocity $u_{s}$ of the spreading flame, which is anticipated to be small compared with $U_{\infty}$, is used with the origin set at the point of inception of vaporization. Upstream of this point the 
surface temperature must rise from $T_{\infty}$ to the vaporization value $T_{v}$ before vaporization can begin, and this requires that part of the heat released at the flame reach this region. In the absence of boundary layer separation, heat must arrive by conduction either through the gas or through the solid, the dominating contribution being the one that carries heat farthest upstream. Counterflow heat conduction in the gas is confined to a Navier-Stokes region at the base of the boundary layer containing the tip of the flame. The characteristic size of this region is $\delta_{N S}=\left(\mu_{g} / \rho_{g} \lambda\right)^{1 / 2}=O\left(\delta_{B} / R e^{1 / 2}\right)$ and the velocity of the fluid in it is of order $u_{N S}=\lambda \delta_{N S}=O\left(U_{\infty} / R e^{1 / 2}\right)$, where $\lambda=\partial u /\left.\partial y\right|_{y=0}=O\left(U_{\infty} / \delta_{B}\right)$ is the shear at the base of the boundary layer immediately upstream of the Navier-Stokes region, $\delta_{B}$ is the displacement thickness of the boundary layer, and $\operatorname{Re}=\rho_{g} U_{\infty} \delta_{B} / \mu_{g} \gg 1$ is the Reynolds number. In the solid, which moves with the unknown velocity $u_{s}$ in the present reference frame, heat conduction against this velocity can reach a region of characteristic size $\delta_{s}=k_{s} / \rho_{s} u_{s} c_{s}$, where $k_{s}, \rho_{s}$ and $c_{s}$ are the conductivity, density and specific heat of the solid.

Counterflow heat conduction in the gas dominates when $\delta_{N S} \gg \delta_{s}$. Then the boundary layer approximation is applicable to the solid, where the energy equation takes the form

$$
\rho_{s} u_{s} c_{s} \frac{\partial T}{\partial x}+m c_{s} \frac{\partial T}{\partial y}=k_{s} \frac{\partial^{2} T}{\partial y^{2}} .
$$

Here $x$ and $y$ are the distances along and normal to the receding solid surface, $m(x)$ is the unknown vaporization flux, and the second term on the left-hand side is the apparent convection due to the recession of the surface. The heat fluxes normal to the surface are of orders $q_{g}=k_{g} \Delta T_{g} / \delta_{N S}$ in the gas and $q_{s}=k_{s} \Delta T_{s} / \delta_{s}$ in the solid, where $\Delta T_{g}=T_{e}-T_{\infty}$ and $\Delta T_{s}=T_{v}-T_{\infty}$ are the scales of the temperature variations in the two phases, $T_{e}$ being the adiabatic flame temperature, and $\delta_{s}$ is now the characteristic thickness of the thermal layer in the solid. The two fluxes are necessarily of the same order (they are equal for $x<0$ ), and this condition determines $\delta_{s}$. The order of $u_{s}$ results then from the balance of the terms $\rho_{s} u_{s} c_{s} \partial T / \partial x=O\left(\rho_{s} u_{s} c_{s} \Delta T_{s} / \delta_{N S}\right)$ and $k_{s} \partial^{2} T / \partial y^{2}=O\left(k_{s} \Delta T_{s} / \delta_{s}^{2}\right)$ in the energy equation (1). These estimates yield the scales

$$
\delta_{s}=\delta_{N S} \frac{k_{s} \Delta T_{s}}{k_{g} \Delta T_{g}} \quad \text { and } \quad u_{s}=u_{N S} \frac{k_{g} \rho_{g} c_{p}}{k_{s} \rho_{s} c_{s}}\left(\frac{\Delta T_{g}}{\Delta T_{s}}\right)^{2}
$$

whereas the ratio of the two terms on the left-hand side of equation (1) is

$$
\frac{m c_{s} \partial T / \partial y}{\rho_{s} u_{s} c_{s} \partial T / \partial x}=O\left(\frac{c_{s} \Delta T_{s}}{L}\right)
$$

where $L$ is the heat of vaporization and use has been made of the relation $m L \sim q_{g}$. The condition that heat conduction in the gas dominate (i.e. $\delta_{N S} \gg \delta_{s}$ ) amounts to $k_{s} \Delta T_{s} / k_{g} \Delta T_{g} \ll 1$.

Assuming, on the other hand, that heat conduction in the solid controls the flame spread, the full Laplacian must be retained on the right-hand side of (1) and the boundary layer approximation can be applied to the high-temperature region of the gas. It can be shown that in the solid all three terms of (1) are equally important, which provides the order of magnitude relations $\rho_{s} u_{s} c_{s}=m c_{s}=k_{s} / \delta_{s}$. In the gas the balance of streamwise convection and transverse molecular transport reads $\rho_{g} u_{g_{0}} / \delta_{s}=\mu_{g} / \delta_{g_{0}}^{2}$, where $\delta_{g_{0}}$ is the thickness of the high-temperature region and $u_{g_{0}}=U_{\infty} \delta_{g_{0}} / \delta_{B}$ is the characteristic velocity in this region. Finally the relation $m L \sim q_{g}$, with $q_{g}=k_{g} \Delta T_{g} / \delta_{g_{0}}$, completes the problem and gives the 
scales

$$
\begin{aligned}
& \delta_{s}=\delta_{N S}\left(\frac{k_{s} \Delta T_{s}}{k_{g} \Delta T_{g}}\right)^{3 / 2}\left(\frac{L}{c_{s} \Delta T_{s}}\right)^{3 / 2} \\
& u_{s}=u_{g_{0}} \frac{k_{g} \rho_{g} c_{p}}{k_{s} \rho_{s} c_{s}}\left(\frac{\Delta T_{g}}{\Delta T_{s}}\right)^{2}\left(\frac{c_{s} \Delta T_{s}}{L}\right)^{2}
\end{aligned}
$$

with $u_{g_{0}}=u_{N S}\left(k_{s} \Delta T_{s} / k_{g} \Delta T_{g}\right)^{1 / 2}\left(L / c_{s} \Delta T_{s}\right)^{1 / 2}$. The ratio of solid-to-gas characteristic fluxes is $q_{s} / q_{g}=c_{s} \Delta T_{s} / L$, so most of the heat flux reaching the surface from the gas is used for vaporization when $c_{s} \Delta T_{s} / L$ is small. The condition for the heat conduction in the solid to dominate is $\delta_{s} \gg \delta_{N S}$, which amounts to $\left(k_{s} \Delta T_{s} / k_{g} \Delta T_{g}\right)\left(L / c_{s} \Delta T_{s}\right) \gg 1$. This condition is the opposite of the one found before for gas conduction dominance if $c_{s} \Delta T_{s} / L=O(1)$, while if this latter quantity is small both gas phase dominance and solid phase dominance are possible in a region of the parameter space, pointing toward possible multiplicity of steady solutions.

The results (2) and (3) give spread rates proportional to the characteristic gas velocity ( $u_{N S}$ and $u_{g_{0}}$, respectively) and hold when the Damköhler number, defined as the ratio of the diffusion time in the Navier-Stokes region $\lambda^{-1}$ to a characteristic chemical time, is large. Effects of finite rate kinetics become important when the Damköhler number is of order unity, leading to a $u_{s}$ that decreases when the characteristic gas velocity increases $[8,9]$, and ultimately to flame extinction when the Damköhler number becomes too small for the flame head to exist in the Navier-Stokes region. An analysis of these finite rate effects, showing how to determine the extinction value of the Damköhler number below which flame spread is not possible, will be presented elsewhere (Fernández-Tarrazo and Liñán, in preparation).

Experiments carried out by Hirano and Kanno [10] with a diffusion flame produced by injecting a gaseous fuel through a porous plate in an air stream parallel to the plate show that the injection and the thermal expansion of the gas lead to an outward displacement of the streamlines around the flame tip, with an associated overpressure which these authors evaluated from their measured velocity and temperature fields. Mao, Kodama and Fernández-Pello [11] numerically solved the Navier-Stokes equations for this configuration and found a pressure maximum ahead of the flame tip and a minimum behind, in agreement with the experimental data. Similar features were later observed for spreading flames; see [12] and [13].

On the other hand, triple-deck theory (e.g., [14]) shows that, at high Reynolds numbers, the outward displacement of the slow flow at the base of the boundary layer is transmitted by the bulk of the boundary layer to the outer irrotational stream, where it generates pressure variations that act back on the slow flow. This occurs in a region of characteristic length $l=R e^{1 / 4} \delta_{B}$ around $x=0$, and the self-induced pressure variations affect the flow in a lower-deck of thickness $\delta_{L D}=\delta_{B} / R e^{1 / 4}$ close up to the solid surface, where $u_{g}=U_{\infty} / R e^{1 / 4}$. These estimates of $l$ and $\delta_{L D}$ result from the convection-pressureviscosity balance $\rho_{g} u_{g}^{2} / l=\Delta p / l=\mu_{g} u_{g} / \delta_{L D}^{2}$ in the lower-deck, with $u_{g}=U_{\infty} \delta_{L D} / \delta_{B}$ and $\Delta p=\rho_{g} U_{\infty}^{2} \delta_{L D} / l$ (from linearized potential theory in the outer flow, where the outward displacement is of order $\delta_{L D}$ ); see [14] for details.

In this paper triple-deck theory is applied to the analysis of flame spread. Generally the lower-deck is much larger than the region determining the flame spread rate $u_{s}$ in the absence of boundary layer separation. This is always the case when conduction in the gas matters (compare $\delta_{N S}$ and $\delta_{s}$ in (2) with the estimates of the previous paragraph) and, typically, also when conduction in the solid dominates ( $\mathrm{cf}(3)$ ). The flow in the lower-deck, 
however, must be analysed in order to evaluate $u_{s}$, because it determines the shear $\lambda$ acting on the small region where counterflow conduction matters (i.e. at $x / l=0^{-}$with the lowerdeck scaling), which is smaller than the shear at the base of the boundary layer upstream of the triple-deck. This analysis is carried out in section 2 for infinitely fast reaction rate (which is a fully justified assumption here, because the local Damköhler number increases as $\left(x / \delta_{N S}\right)^{2 / 3}$ downstream of the Navier-Stokes region, where it must be at least of order unity in order for a flame to exist, and therefore is of $O\left(R e^{1 / 2}\right)$ or larger in the lowerdeck), whereas a detailed description of the flow, temperature and concentration fields in the region of size $\delta_{N S}$ or $\delta_{s}$, required to calculate $u_{s}$, will be given elsewhere. The pressure gradient induced by the vaporization and the thermal expansion of the gas is found to be adverse for $x<0$, in agreement with $[10,11,12,13]$. This pressure gradient may lead to local separation of the boundary layer at a point on the solid surface upstream of the vaporizing region and, when this happens, heat transfer by upstream convection in the region of reverse flow, of size $O(l)$, is enabled. This is a more efficient heat transfer mechanism than counterflow conduction in either phase, resulting in flame spread rates much higher than predicted by any of the estimations (2) or (3), and fully determined now by the flow in the lower-deck. Self-induced boundary layer separation is discussed in section 3.

\section{Triple-deck problem without separation}

The boundary layer approximation is applicable to both phases in the triple-deck region, which greatly simplifies the analytical treatment. In what follows a subscript 0 will be added to all the magnitudes pertaining to the small region around $x=0$ determining $u_{s}$, and $l_{0}$ will denote the length of this region, which is equal to $\delta_{N S}$ if heat conduction in the gas matters and to $\delta_{s}$ given by (3) if heat conduction in the solid dominates. The estimations resulting from the balance of convection and viscous forces in the lowerdeck of the gas can then be recast as $u_{g}=u_{g_{0}}\left(\delta_{L D} / \delta_{g_{0}}\right)$ and $\delta_{L D}=\delta_{g_{0}}\left(l / l_{0}\right)^{1 / 3}$, leading to $q_{g}=q_{g_{0}}\left(l_{0} / l\right)^{1 / 3}$ in this region and, since this heat flux is responsible for the vaporization of the solid, $m / m_{0}=\left(l_{0} / l\right)^{1 / 3}$. Owing to the slow decay of the vaporization flux, the second term on the left-hand side of (1) ends up dominating over the first term, which leads to the simplified equation $m c_{s} \partial T / \partial y=k_{s} \partial^{2} T / \partial y^{2}$, whose solution with the boundary conditions $T=T_{v}$ at $y=0$ and $T=T_{\infty}$ for $y \rightarrow-\infty$ gives $q_{s} \equiv k_{s}(\partial T / \partial y)_{y=0}=m c_{s}\left(T_{v}-T_{\infty}\right)$ for $x>0$, whereas $T=T_{\infty}$ for $x<0$. To verify the consistency of this simplification notice that, from the foregoing estimations, $\left(\rho_{s} u_{s} c_{s} \partial T / \partial x\right) /\left(m c_{s} \partial T / \partial y\right)=O\left[\left(q_{s_{0}} / q_{g_{0}}\right)\left(L / c_{s} \Delta T_{s}\right)\right]^{2} O\left[\left(l_{0} / l\right)^{1 / 3}\right]$. The first of these two factors is of order unity or small except when heat conduction in the gas dominates and $c_{s} \Delta T_{s} / L$ is small, in which case the first term of (1) is much larger than the second in the Navier-Stokes region and both become comparable in an intermediate region before the second term dominates. The second factor is of order $R e^{-1 / 4} \ll 1$ when heat conduction in the gas matters and somewhat less small when heat conduction in the solid dominates.

If the Lewis numbers of the two reactants are equal to unity, as will be assumed in the remainder of this paper, the Shvab-Zeldovich variables

$$
Z=\frac{S Y_{F}-Y_{O}+1}{1+S} \quad \text { and } \quad H=T-1+\gamma\left(Y_{F}+Y_{O}-1\right)
$$

which are transported as passive scalars, can be introduced. Here $Y_{O}$ and $Y_{F}$ are the mass fractions of oxygen and fuel, the first one scaled with its free stream value, and $T$ is the temperature scaled with $T_{\infty}$. The parameters $S$ and $\gamma$ are the air-to-fuel mass stoichiometric ratio and a measure of the exothermicity of the gas-phase reaction, $\gamma=\left(Q / c_{p} T_{\infty}\right) /(1+S)$, 
respectively, where $Q$ is the heat released by the gas phase reaction per unit mass of fuel. The variables (4) are the mixture fraction and the excess of enthalpy (thermal plus formation) over its free stream value (here $H<0$ ). In the Burke-Schumann limit, the additional relation $Y_{F} Y_{O}=0$ is satisfied because the oxygen and the fuel do not coexist outside the infinitely thin flame sheet, and the temperature and the reactant mass fractions are piecewise linear functions of $Z$ and $H$ :

$$
\begin{aligned}
& Y_{F}=0, \quad Y_{O}=1-\frac{Z}{Z_{s}}, \quad T=1+H+\gamma \frac{Z}{Z_{s}} \quad \text { for } \quad 0 \leqslant Z \leqslant Z_{s} \\
& Y_{F}=\frac{Z-Z_{s}}{1-Z_{s}}, \quad Y_{O}=0, \quad T=1+H+\gamma \frac{1-Z}{1-Z_{s}} \quad \text { for } \quad Z_{s} \leqslant Z \leqslant 1
\end{aligned}
$$

where $Z_{s}=1 /(1+S)$ is the value of $Z$ at the flame.

Appropriate scaling factors for the streamwise and transverse distances, the corresponding components of the velocity, and the pressure variations in the lowerdeck of the gas are $\delta_{B} R e^{1 / 4} / \Lambda^{5 / 4}, \delta_{B} / R e^{1 / 4} \Lambda^{3 / 4}, U_{\infty} \Lambda^{1 / 4} / R e^{1 / 4}, U_{\infty} \Lambda^{3 / 4} / R e^{3 / 4}$, and $\rho_{g} U_{\infty}^{2} \Lambda^{1 / 2} / R e^{1 / 2}$, respectively, where $\Lambda=\left(\delta_{B} / U_{\infty}\right) \partial u /\left.\partial y\right|_{0}=O(1)$ is the nondimensional shear at the base of the boundary layer upstream of the triple-deck $(\Lambda \approx 0.57$ for Blasius boundary layer). Assuming, in addition, that the viscosity increases as a power of the temperature, $\mu \propto T^{\sigma}$, and scaling the density and viscosity with their free stream values $\rho_{g}$ and $\mu_{g}$, the governing equations for the gas take the form

$$
\begin{aligned}
& \nabla \cdot(\rho v)=0 \\
& \rho v \cdot \nabla u=-\frac{\mathrm{d} p}{\mathrm{~d} x}+\frac{\partial}{\partial y}\left(T^{\sigma} \frac{\partial u}{\partial y}\right) \\
& \rho v \cdot \nabla Z=\frac{1}{\operatorname{Pr}} \frac{\partial}{\partial y}\left(T^{\sigma} \frac{\partial Z}{\partial y}\right) \\
& \rho v \cdot \nabla H=\frac{1}{\operatorname{Pr}} \frac{\partial}{\partial y}\left(T^{\sigma} \frac{\partial H}{\partial y}\right) \\
& \rho T=1 \\
& p=-\frac{1}{\pi} \int_{-\infty}^{\infty} \frac{\mathrm{d} A / \mathrm{d} x^{\prime}}{x-x^{\prime}} \mathrm{d} x^{\prime}
\end{aligned}
$$

while the boundary conditions expressing the matching with the effectively inviscid flow in the bulk of the boundary layer above the lower-deck and with the oncoming unperturbed boundary layer are

$$
\begin{aligned}
& u=y-A(x), \quad Z=H=0 \quad \text { for } y \rightarrow \infty \\
& u-y=Z=H=0 \quad \text { for } x \rightarrow-\infty .
\end{aligned}
$$

Here $v=(u, v), \operatorname{Pr}$ is the Prandtl number, assumed to be constant, compressibility effects have been left out of the equation of state $(6 e)$, and $(6 f)$ is the nonlocal relation between the unknown outward displacement of the outer flow $A(x)$ and the pressure variation it induces. This relation, where the principal part of the integral is understood, comes from linearized potential theory, and makes the problem elliptic despite the boundary layer approximation in $(6 b)$ to $(6 d)$ (see [14] for details).

Boundary conditions for $Z$ and $H$ at the solid surface can be obtained by linearly combining the conservation conditions for the fuel $\left(m Y_{F}-\left(T^{\sigma} / P r\right) \partial Y_{F} / \partial y=m\right)$, the oxygen $\left(m Y_{O}-\left(T^{\sigma} / P r\right) \partial Y_{O} / \partial y=0\right)$, and the energy $\left(\left(T^{\sigma} / P r\right) \partial T / \partial y=m L+q_{s}=\right.$ $\left.m\left[L+\left(c_{s} / c_{p}\right)\left(T_{v}-1\right)\right]\right)$ across the surface. Here the vaporization flux $m(x)$ is scaled with $\rho_{g} U_{\infty} \Lambda^{3 / 4} / R e^{3 / 4}$, the heat of vaporization $L$ is scaled with $c_{p} T_{\infty}$, the vaporization 
temperature is scaled with $T_{\infty}$, and use has been made of the expression of the heat flux entering the solid obtained in the first paragraph of this section to write the last term of the latter equation. The resulting boundary conditions are

$$
m Z-\frac{T^{\sigma}}{\operatorname{Pr}} \frac{\partial Z}{\partial y}=m \quad \text { and } \quad m H-\frac{T^{\sigma}}{\operatorname{Pr}} \frac{\partial H}{\partial y}=-m \tilde{L} \quad \text { at } \quad y=0
$$

where $\tilde{L}=L+\left(c_{s} / c_{p}-1\right)\left(T_{v}-1\right)$. Equations $(6 c)$ and $(6 d)$ with these boundary conditions yield $H=-\tilde{L} Z$, so $H$ need not be computed separately of $Z$. Using this result, the temperature in (5) can be written in terms of the mixture fraction only. In particular, the temperature of the flame $\left(Z=Z_{s}\right)$ is constant, equal to $T_{f}=1+\gamma-\tilde{L} Z_{s}$, where the last term is the difference with the adiabatic flame temperature, $T_{e}=1+\gamma$, due to the heat required to heat up and vaporize the solid. Also, $Z=0$ for $x<0$ in the absence of separation.

The rest of the boundary conditions at the surface are

$$
y=0:\left\{\begin{array}{c}
u=0, \quad \rho v=m \\
m Z-\frac{T^{o}}{P r} \frac{\partial Z}{\partial y}=m, \quad Z=Z_{v} \\
u=v=Z=m=0
\end{array} \quad \text { for } \quad x>0\right.
$$

where $Z_{v}$ is the value of $Z$ obtained from (5) with $T=T_{v}$. Two possibilities exist. Either $Z_{v}>Z_{s}$, and then the second line of (5) must be used, giving $Z_{v}=\left[\gamma-\left(1-Z_{s}\right)\left(T_{v}-\right.\right.$ $1)] /\left[\gamma+\left(1-Z_{s}\right) \tilde{L}\right]$, which is greater than $Z_{s}$ for $\gamma>\gamma_{\min }=Z_{s} \tilde{L}+T_{v}-1$; or $Z_{v}<Z_{s}$, and then the first line of (5) must be used, giving $Z_{v}=Z_{s}\left(T_{v}-1\right) /\left(\gamma-Z_{s} \tilde{L}\right)$, which is smaller than $Z_{s}$ also for $\gamma>\gamma_{\min }$. Thus, two solutions exist for $\gamma$ above $\gamma_{\min }$, coinciding with each other when $\gamma=\gamma_{\min }$, and no solution exists for $\gamma<\gamma_{\min }$, for which the reaction is not sufficiently exothermic to keep the flame spreading steadily. The solution with $Z_{v}<Z_{s}$ has the flame lying on the relatively cold solid surface, which might invalidate the assumption of infinitely fast kinetics in realistic cases with large activation energy reactions. In addition this solution is probably unstable.

The problem $(6 a)-(6 c)$ and $(6 e)-(6 i)$ contains six non-dimensional parameters: $\gamma, S, \tilde{L}$, $T_{v}, \sigma$ and $\mathrm{Pr}$. Its solution, which must be obtained numerically, determines in particular the non-dimensional shear $\lambda=\partial u / \partial y\left(x=0^{-}, y=0\right)<1$ acting on the inner region discussed in the previous section.

For $x \gg 1$ the effect of the pressure gradient becomes negligible in $(6 b)$ and the solution takes on the self-similar Goldstein's form [15]

$$
\psi=x^{2 / 3} f_{G}(\eta), \quad m=\phi_{G} / x^{1 / 3}, \quad Z=Z_{G}(\eta), \quad \eta=y / x^{1 / 3}
$$

where $\psi$ is the stream function $(\rho u=\partial \psi / \partial y, \rho v=-\partial \psi / \partial x$ and $\psi=0$ at the solid surface for $x<0$ ) and

$$
\begin{aligned}
& {\left[T^{\sigma}\left(T f_{G}^{\prime}\right)^{\prime}\right]^{\prime}+\frac{2}{3} f_{G}\left(T f_{G}^{\prime}\right)^{\prime}-\frac{1}{3} T f_{G}^{\prime 2}=0} \\
& \left(T^{\sigma} Z_{G}^{\prime}\right)^{\prime}+\frac{2}{3} \operatorname{Pr} f_{G} Z_{G}^{\prime}=0 \\
& \eta=0:\left\{\begin{array}{lc}
f_{G}=-\frac{2}{3} \phi_{G} & f_{G}^{\prime}=0 \\
Z_{G}=Z_{v} & Z_{G}^{\prime}=-\operatorname{Pr}\left(1-Z_{v}\right) \phi_{G} / T_{v}^{\sigma}
\end{array}\right. \\
& \eta \rightarrow \infty: f_{G}^{\prime \prime}=1 \quad Z_{G}=0
\end{aligned}
$$

primes denoting derivatives with respect to $\eta$. The solution of the problem (8) determines $\phi_{G}\left(\gamma, S, \tilde{L}, T_{v}, \sigma, P r\right)$. The outward displacement is here $A=a x^{1 / 3}$, where $a=$ 
$\lim _{\eta \rightarrow \infty}\left(\eta-f_{G}^{\prime}\right)$, leading via $(6 f)$ to a pressure variation $-(a / 3 \sqrt{3}) / x^{2 / 3}$ for $x \gg 1$ and $(2 a / 3 \sqrt{3}) /(-x)^{2 / 3}$ for $(-x) \gg 1$.

For $0<x \ll 1$ the thickness of the layer of warm gas grows as $x^{1 / 3}$ in the surrounding cold gas where $u=\lambda y$. An outward displacement of the outer stream proportional to $x^{1 / 3}$ is now inadmissible because (6f) would then give a diverging pressure. Consistency, therefore, requires that the layer of warm gas grow without displacing the velocity profile of the cold flow, and this in turn requires a pressure decrease proportional to $x^{2 / 3}$ whose gradient accelerates the warm gas compensating its tendency to open up. The solution in the layer of warm gas is then of the Hakkinen-Rott form [16]

$$
\begin{array}{lr}
\psi=\lambda^{1 / 3} x^{2 / 3} f_{H R}(\xi) & m=\lambda^{1 / 3} \phi_{H R} / x^{1 / 3} \\
p-p(0)=-\lambda^{4 / 3} b x^{2 / 3} & Z=Z_{H R}(\xi) \\
\text { with } \quad \xi=\lambda^{1 / 3} y / x^{1 / 3} &
\end{array}
$$

where $f_{H R}, Z_{H R}$ and $\phi_{H R}$ satisfy $(8 a)-(8 c)$ with the extra term $-\frac{2}{3} b$ added to the right-hand side of $(8 a)$ and the conditions of matching to the outer flow without additional displacement

$$
f_{H R}=\frac{1}{2} \xi^{2}+o(\xi) \quad \text { and } \quad Z_{H R}=0 \quad \text { for } \quad \xi \rightarrow \infty
$$

instead of $(8 d)$. Conditions $\left(8 d^{\prime}\right)$ are stronger than conditions $(8 d)$ and also determine $b\left(\gamma, S, \tilde{L}, T_{v}, \sigma, P r\right)$.

The coefficients $a$ and $b$ of the outward displacement in Goldstein's region and of the pressure gradient required to prevent any displacement in the Hakkinen-Rott region, along with the coefficients $\phi_{G}$ and $\phi_{H R}$ of the corresponding vaporization fluxes, are given in figure 1 as functions of $\gamma$ for $\sigma=P r=1$ and the values $S=8, T_{v}=2.24$ and $\tilde{L}=3.29$ corresponding to PMMA in air (taken from [17]). The flame is separated from the surface in the upper branch of figure 1 , on which the scaled velocity profiles $\left(T\left(Z_{H R}\right) f_{H R}^{\prime}\right.$, not shown) present a local maximum at a finite $\xi$ when $\gamma$ is larger than about 3 , due to the action of the favourable gradient of the pressure in (9) on the light high-temperature gas surrounding the flame. This is in agreement with the experimental results of [10] and others.

For the same values of $S, \tilde{L}, T_{v}, \sigma$ and $\operatorname{Pr}$, figure 2 shows $\lambda(\gamma)$ obtained from the numerical solution of the problem $(6 a)-(6 c)$ and $(6 e)-(6 i)$. In this figure the upper branch corresponds to solutions with the flame lying on the surface, for which the vaporization flux decreases with increasing $\gamma$ and tends to zero as $1 / \gamma$ for $\gamma \rightarrow \infty$. In this limit one is left with the pure heat transfer problem of a gas stream parallel to an inert surface whose temperature is 1 (ambient) for $x<0$ and $T_{v}$ for $x>0$. This problem has been analysed elsewhere [18], giving in particular $\lambda \approx 0.622$ for $T_{v}=2.24$. In the lower branch of figure 2 , corresponding to solutions with the flame separated from the surface, $\lambda$ decreases with increasing $\gamma$ and becomes equal to zero for $\gamma \approx 3.7$, which defines the onset of boundary layer separation over the non-vaporizing region of the solid. The numerical solutions also show that, for any $\gamma$, the pressure increases in the streamwise direction over the non-vaporizing part of the solid (and the skin friction decreases accordingly), attains a maximum at $x=0$, and then rapidly decreases (with an infinite slope for $x \searrow 0$; cf (9)), reaching a minimum smaller than the ambient pressure at a point on the vaporizing part of the solid and tending to the ambient pressure from below as $x \rightarrow \infty$. All this is in line with the experimental results of Hirano and Kanno [10] but, contrarily to the findings of [11], the extremes of the pressure are not due to upstream conduction, which is absent in the triple-deck region. 


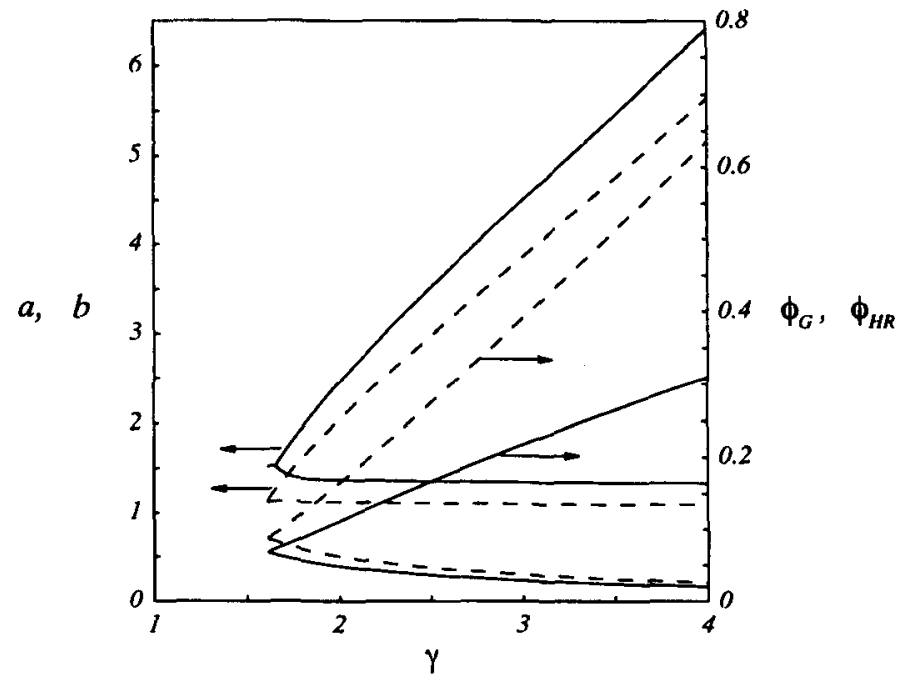

Figure 1. Coefficients $a$ and $\phi_{G}$ of Goldstein's solution (7) (solid), and $b$ and $\phi_{H R}$ of the Hakkinen-Rott solution (9) (dashed), as functions of $\gamma$. The flame lies on the surface in the lower branch solutions.

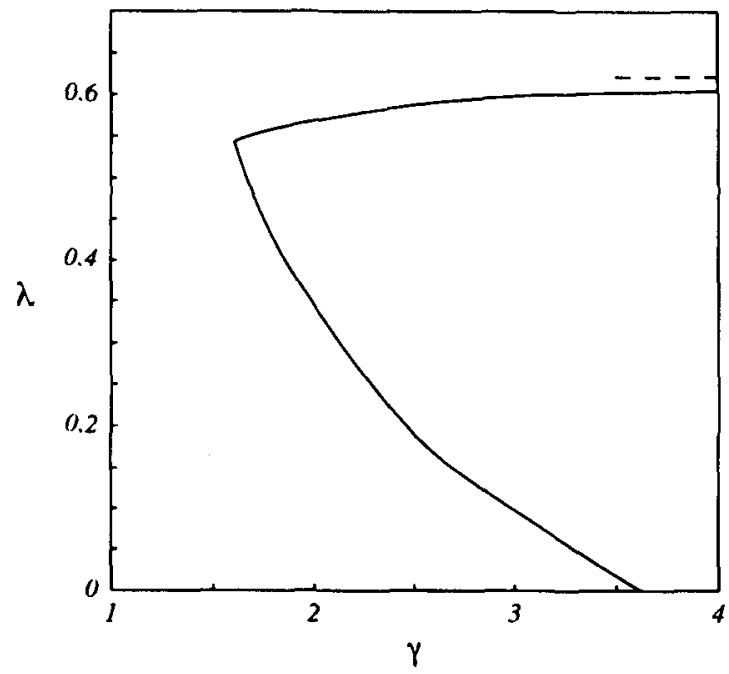

Figure 2. Non-dimensional shear at $\left(x=0^{-}, y=0\right)$, acting on the inner region determining $u_{s}$, as a function of $\gamma$. The flame lies on the surface in the upper branch solutions.

\section{Flame spread with boundary layer separation}

The mechanism of heat transfer from the flame to the solid surface, and the order of the flame spread rate, change when the boundary layer separates under the action of the adverse pressure gradient induced by the vaporization and the thermal expansion of the gas. Then a region of reverse flow appears on the non-vaporizing part of the solid, and the tip of 
the flame gets into the recirculating flow, because some unburned fuel vapour enters this region (see inset of figure 3). Furthermore, the heat released by the flame is convected upstream over the whole extent of the recirculation bubble, whose length scales with the length $l=R e^{1 / 4} \delta_{B}$ of the triple-deck. To estimate the order of the flame spread rate under these conditions, note, coming back to dimensional variables, that the heat flux reaching the surface and entering the solid in the recirculating flow is of order $k_{g} \Delta T_{g} / \delta_{L D}=k_{s} \Delta T_{s} / \delta_{s}$, whence the thickness of the thermal layer in the solid must be $\delta_{s}=\delta_{L D}\left(k_{s} \Delta T_{s}\right) /\left(k_{g} \Delta T_{g}\right)$. Using this result, the balance of convection and conduction in the energy equation (1) for the solid (i.e., $\rho_{s} u_{s} c_{s} / l=k_{s} / \delta_{s}^{2}$ ) yields the new scale of the flame spread velocity

$$
u_{g} \frac{k_{g} \rho_{g} c_{p}}{k_{s} \rho_{s} c_{s}}\left(\frac{\Delta T_{g}}{\Delta T_{s}}\right)^{2}\left(\frac{c_{s} \Delta T_{s}}{L}\right)^{2}
$$

where $u_{g}=\Lambda^{1 / 4} U_{\infty} / R e^{1 / 4}$ is the scale of the gas velocity in the lower deck, much larger by a factor $R e^{1 / 4}$ than $u_{N S}$ in (2) and also typically much larger than $u_{g_{0}}$ in (3).

As in the case of flame spread controlled by heat conduction in the gas,

$$
\frac{m c_{s} \partial T / \partial y}{\rho_{s} u_{s} c_{s} \partial T / \partial x}=O\left(\frac{c_{s} \Delta T_{s}}{L}\right) \text {. }
$$

In this section the assumption $c_{s} \Delta T_{s} / L \ll 1$ will be made in order to simplify the analysis of the solid neglecting the second term on the left hand side of (1). Using again the non-dimensional variables introduced in the previous section, and scaling the solid temperature with $T_{\infty}, u_{s}$ with (10) and the distance in the solid normal to the surface with $\left(\delta_{B} / \operatorname{Re}^{1 / 4} \Lambda^{3 / 4}\right)\left(k_{s} \Delta T_{s}\right) /\left(k_{g} \Delta T_{g}\right)$, the simplified energy equation (1) becomes $(1+\gamma)^{2} /\left(T_{v}-1\right)^{2} u_{s} \partial T / \partial x=\partial^{2} T / \partial y^{2}$, for which the relations

$$
q_{s}(x)=\frac{1+\gamma}{T_{v}-1} \sqrt{\frac{u_{s}}{\pi}} \int_{-\infty}^{x} \frac{\partial T\left(x_{1}, 0\right) / \partial x_{1}}{\left(x-x_{1}\right)^{1 / 2}} \mathrm{~d} x_{1}
$$

and

$$
T(x, 0)-1=\frac{T_{v}-1}{1+\gamma} \frac{2}{\sqrt{u_{s} \pi}} \int_{-\infty}^{x}\left(x-x_{1}\right)^{1 / 2} \frac{\mathrm{d} q_{s}}{\mathrm{~d} x_{1}} \mathrm{~d} x_{1}
$$

hold between the surface temperature and the heat flux entering the solid (see, e.g., [19]). Here the upper limit of the first integral can be set equal to zero when computing $q_{s}(x)$ for $x>0$, because the temperature of the vaporizing surface is uniform. In the second relation $q_{s}$ is equal to $q_{g}$ for $x<0$. In particular, imposing that $T(0,0)=T_{v}$,

$$
u_{s}=\frac{4}{\pi(1+\gamma)^{2}}\left\{\int_{-\infty}^{0}(-x)^{1 / 2} \frac{\mathrm{d} q_{g}}{\mathrm{~d} x} \mathrm{~d} x\right\}^{2} \text {. }
$$

Finally, eliminating $T(x, 0)$,

$$
q_{s}(x)=\frac{x^{1 / 2}}{\pi} \int_{-\infty}^{0} \frac{q_{g}\left(x_{1}\right) \mathrm{d} x_{1}}{\left(-x_{1}\right)^{1 / 2}\left(x-x_{1}\right)} \quad \text { for } \quad x>0 .
$$

In non-dimensional variables, the condition $c_{s} \Delta T_{s} / L \ll 1$ amounts to $\left(T_{v}-1\right)$ small or $\gamma$ large. In either case non-dimensional temperature variations of order $\left(T_{v}-1\right)$ are negligible in the gas. Using this condition and $q_{s}$ given by (12), the boundary conditions at the solid surface become

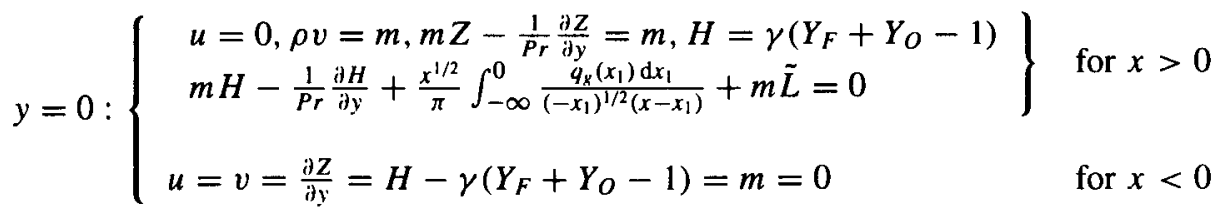


where $q_{g}(x)=(1 / P r) \partial T /\left.\partial y\right|_{0}$ and $Y_{F}(Z), Y_{O}(Z)$ and $T(Z, H)$ are given by (5).

The solution of the problem $(6 a)-(6 h)$ and $\left(6 i^{\prime}\right)$ determines the velocity, pressure, temperature and reactant mass fractions in the gas, and the vaporization flux. The flame spread rate can then be evaluated from (11). The resulting $u_{s}(\gamma)$ and the distance $-x_{s}$ from the separation point to the onset of vaporization are given in figure 3 for the values of $S, \tilde{L}$, $\sigma$ and $\operatorname{Pr}$ used in the computations of the previous section. The small numerical values of $u_{s}$ are a consequence of the small velocity of the recirculating gas. As can be seen, the results of figure 3 show that boundary layer separation occurs for $\gamma>\gamma_{c} \approx 4.3$, which is greater than the value 3.7 obtained in the previous section. The difference is due to the simplifications made in $\left(6 i^{\prime}\right)$, where terms of order $\left(T_{v}-1\right)$ have been neglected in the boundary conditions containing the excess of enthalpy $H$. The result of the previous section is recovered if the computations are repeated with the full expression $H=T-1+\gamma\left(Y_{F}+Y_{O}-1\right)$ retained in $\left(6 i^{\prime}\right)$ and the gas conductivity evaluated as $T^{\sigma} / P r$ at the surface, which is consistent with the present approximation. Some results obtained with this modified form of $\left(6 i^{\prime}\right)$ are included in figure 3 (thin curves).

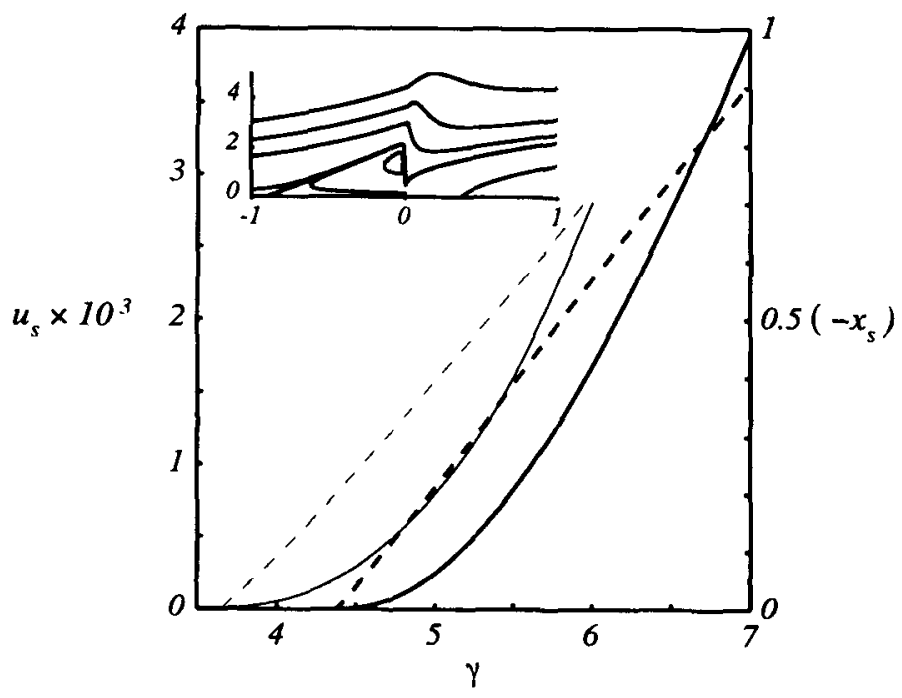

Figure 3. Thick curves: non-dimensional $u_{s}$ (solid) and position of the separation point (dashed) as functions of $\gamma$ for $S=8, T_{v}=2.24, \tilde{L}=3.29$ and $\sigma=\operatorname{Pr}=1$. The thin curves are for the modified form of the boundary conditions $\left(6 i^{\prime}\right)$ commented on in the text. Inset: streamlines $\psi=0, \pm 0.0015, \pm 0.5,0.18,1.13$ and -0.025 (in the recirculation region only) for $\gamma=7$.

The structure of the solution around $x=0$ for values of $\gamma$ slightly above the onset of separation $\left(\epsilon \equiv\left(\gamma-\gamma_{c}\right) \ll 1\right)$ is of some interest, and will be analysed here following the lines of [20] and [18]. The numerical solution of $(6 a)-(6 h)$ and $\left(6 i^{\prime}\right)$ shows that the size of the bubble begins increasing linearly with $\epsilon$ for small values of this parameter, and that the shear on the solid (not displayed) varies linearly with $x$ in the region of the bubble: $\partial u /\left.\partial y\right|_{y=0}=s_{0}\left(x_{s}-x\right)$ for $x=O\left(x_{s}\right)$, where $x_{s}=O(\epsilon)<0$ is the separation point. The balance of viscous and pressure forces in this region, where $p_{0}^{\prime} \equiv \mathrm{d} p /\left.\mathrm{d} x\right|_{x=0^{-}}=O(1)$ in $(6 b)$, then requires $u=O\left(\epsilon^{2}\right)$, implying that the convective terms are negligible in the bulk of the bubble. (The values of $s_{0}, p_{0}^{\prime}$, and $x_{s} / \epsilon$ depend on the flow over the whole lower-deck). The solution of the simplified momentum equation with the no-slip condition 
$\left(6 i^{\prime}\right)$ at the solid surface is $u=\frac{1}{2} p_{0}^{\prime} y^{2}+s_{0}\left(x_{s}-x\right) y$, leading to $y=3\left(s_{0} / p_{0}^{\prime}\right)\left(x-x_{s}\right)$ on the dividing streamline. Analogously, $(6 c)$ and $(6 d)$ imply $Z=H=0$ in the bulk of the bubble.

The effect of the convection reappears in a short region where $x=O\left(\epsilon^{4}\right)$ and $\rho v \cdot \nabla u=O\left[\partial\left(T^{\sigma} \partial u / \partial y\right) / \partial y\right]=O(1)$. This is the region of penetration of the fuel vapour and the flame over the non-vaporizing part of the solid, where the gas expands on receiving heat from the flame. The pressure gradient in this region is also $O(1)$ and changes from adverse to favourable for the reason already mentioned in the previous section (cf paragraph above equation (9)); namely, that in the absence of the appropriate $O(1)$ pressure gradient the thermal expansion of the gas would lead to a displacement of order $\epsilon$ of the outer velocity profile, resulting, via (6f), in a much larger pressure gradient of order $\epsilon^{-7}$, too strong to be accommodated by the local flow. Appropriate rescaled variables to describe this region are $\tilde{x}=p_{0}^{\prime 3} x / 8 s_{0}^{4} x_{s}^{4}, \tilde{y}=p_{0}^{\prime} y / 2 s_{0}\left(-x_{s}\right), \tilde{u}=p_{0}^{\prime} u / 2 s_{0}^{2} x_{s}^{2},(\tilde{v}, \tilde{m})=2 s_{0}\left(-x_{s}\right)(v, m) / p_{0}^{\prime}$, and $\tilde{p}=p_{0}^{\prime 2}(p-p(0)) / 4 s_{0}^{4} x_{s}^{4}$. Equations $(6 a)-(6 e)$ and $\left(6 i^{\prime}\right)$ (with $\left.\gamma=\gamma_{c}\right)$ are left invariant by this rescaling, and are to be solved with the additional conditions

$$
\tilde{u}=-\tilde{y}+\tilde{y}^{2}+o(1), \quad Z=H=0 \quad \text { for } \quad \tilde{y} \rightarrow \infty
$$

and

$$
\tilde{u}=-\tilde{y}+\tilde{y}^{2}, \quad Z=H=0, \quad \frac{\mathrm{d} \tilde{p}}{\mathrm{~d} \tilde{x}}=2 \quad \text { for } \quad \tilde{x} \rightarrow-\infty
$$

which are the matching conditions with the region above the bubble (with the requirement of zero displacement) and with the transport-dominated bulk of the recirculating flow described above, respectively.

Some streamlines and isotherms, as well as the flame sheet, from the numerical solution of $(6 a)-(6 e),\left(6 g^{\prime}\right)-\left(6 h^{\prime}\right)$ and $\left(6 i^{\prime}\right)$ for the values of the parameters used in the previous cases are displayed in figure $4(a)$, and the distributions of pressure, heat flux from the gas to the surface, and vaporization flux are given in figure $4(b)$. The computed flame spread rate is $0.015 s_{0}^{2} x_{s}^{2} / p_{0}^{\prime} \approx 7.4 \times 10^{-4} \epsilon^{2}$, where use has been made of the results $s_{0} \approx 0.462$, $p_{0}^{\prime} \approx 0.261$ and $x_{s} / \epsilon \approx-0.247$ to write the second equality.

It may be worth noting that the foremost point of the flame is here the flame tail rather than the flame head, in the sense that the fluid at that point moves in the upstream direction. While the flame always ends at the solid surface in the present infinitely fast reaction analysis, the distinction is important for finite rate kinetics, for which a quench region exists on top of the cold surface. Now extinction of the flame in this region brings only minor changes to the present solution, not the total breakdown that occurs when the triple flame making the head of a flame in forward flow can no longer exist in the tiny Navier-Stokes region.

The heat flux entering the solid becomes negligible in the energy balance at the surface in $\left(6 i^{\prime}\right)$ when $\tilde{x} \rightarrow \infty$, so the result $H=-\tilde{L} Z$ of the previous section is recovered in this limit. In addition, the term $\tilde{y}^{2}$ dominates in the boundary condition $\left(6 g^{\prime}\right)$ for the velocity, and the solution takes the self-similar form

$$
\begin{aligned}
& \tilde{\psi}=\tilde{x}^{3 / 4} f_{1}(\zeta), \quad \tilde{m}=\phi_{1} / \tilde{x}^{1 / 4}, \quad \tilde{p}=-c \tilde{x}, \quad Z=Z_{1}(\zeta) \\
& \text { with } \quad \zeta=\tilde{y} / \tilde{x}^{1 / 4}
\end{aligned}
$$

where

$$
\begin{aligned}
& {\left[T_{1}^{\sigma}\left(T_{1} f_{1}^{\prime}\right)^{\prime}\right]^{\prime}+\frac{3}{4} f_{1}\left(T_{1} f_{1}^{\prime}\right)^{\prime}-\frac{1}{2} T_{1} f_{1}^{\prime 2}=-c} \\
& \left(T_{1}^{\sigma} Z_{1}^{\prime}\right)^{\prime}+\frac{3}{4} \operatorname{Pr} f_{1} Z_{1}^{\prime}=0
\end{aligned}
$$



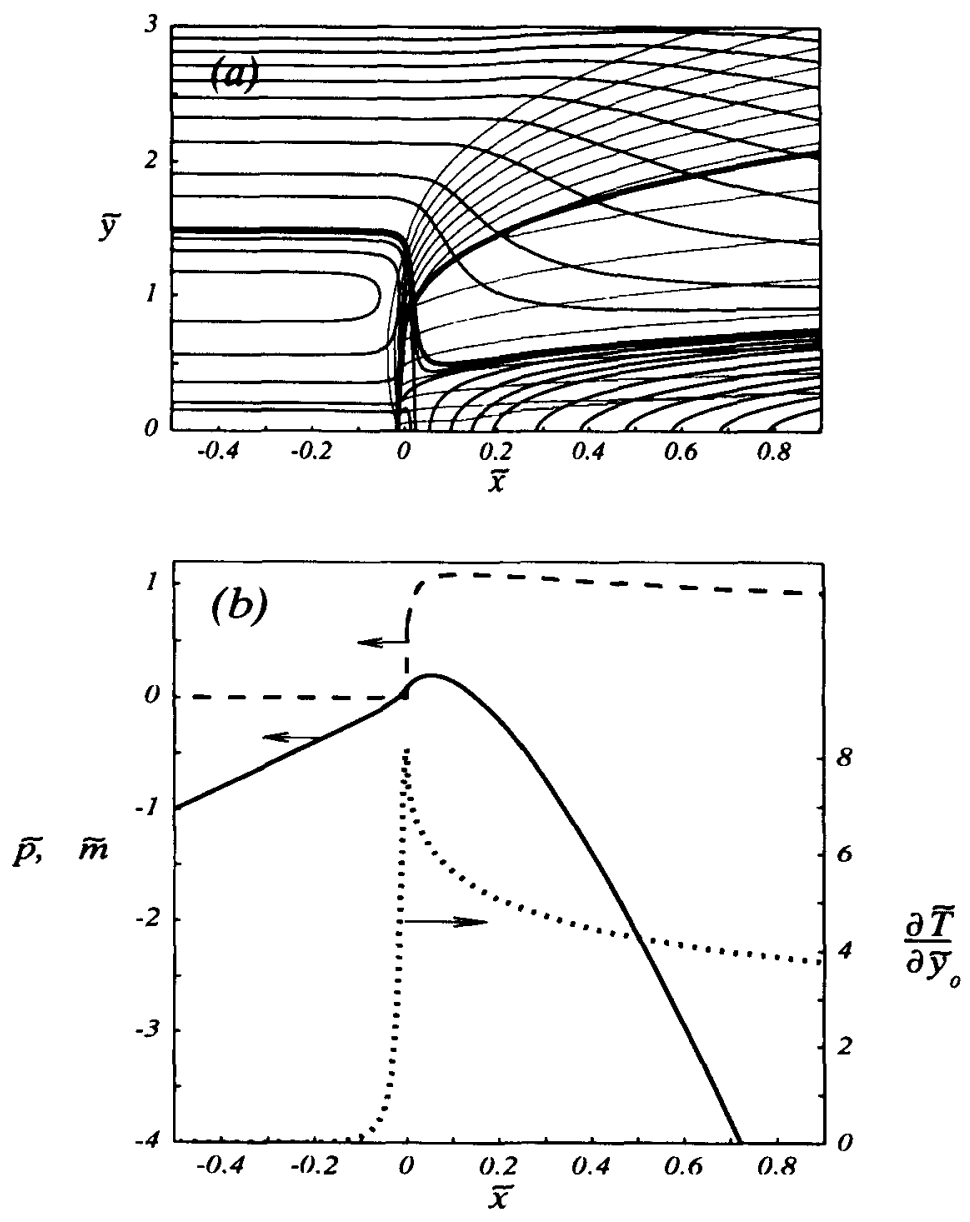

Figure 4. (a) Streamlines $\psi=-0.9$ to 0 (step 0.1 ), -0.15 to 0 (step 0.05 ), -0.0184 (boundary of the recirculation bubble), $-0.01,0.25$, and 0 to 4 (step 0.5 ); the streamline $\psi=0$ is thicker than the others. Isotherms 1.4 to 4.2 (step 0.4); thin curves. Flame sheet; thickest curve. (b) Distributions of pressure (solid), vaporization flux (dashed), and heat flux from the gas to the surface (dotted).

$$
\begin{aligned}
& \zeta=0:\left\{\begin{array}{l}
f_{1}=-\frac{4}{3} \phi_{1} \quad f_{1}^{\prime}=0 \\
Z_{1}^{\prime}+\operatorname{Pr} \phi_{1}\left(1-Z_{1}\right)=0 \quad \gamma_{c}\left(Y_{F}+Y_{O}-1\right)+\tilde{L} Z_{1}=0
\end{array}\right. \\
& \zeta \rightarrow \infty: \quad f_{1}=\frac{1}{3} \zeta^{3}+o\left(\zeta^{2}\right) \quad Z_{1}=1
\end{aligned}
$$

primes denoting now derivatives with respect to $\zeta$. The solution of this problem determines $f_{1}, Z_{1}, c$ and $\phi_{1}$. In particular, $c \approx 22.809$ and $\phi_{1} \approx 1.304$ for the values of the parameters used before. 


\section{Conclusions}

The viscous-inviscid interaction due to the vaporization and the thermal expansion of the gas around the tip of a flame spreading against an opposing forced flow has been analysed. The triple-deck problem has been formulated and numerically solved, showing that an adverse self-induced pressure gradient appears that can lead to separation of the boundary layer on the non-vaporizing part of the solid, which results in a drastic change in the upstream heat transfer mechanism and the flame spread rate.

In the absence of boundary layer separation the region of counterflow conduction determining the flame spread rate is much smaller than the lower-deck of the interaction region and is embedded in it. The adverse pressure gradient induced by the interaction on the non-vaporizing part of the solid reduces the shear $\lambda$ acting on that small region from its unperturbed value farther upstream, and therefore decreases the flame spread rate in the heat-transfer regime (cf (2) and (3), where $u_{N S} \propto \lambda^{1 / 2}$ ).

When the boundary layer separates, the foremost point of the flame is in the region of reverse flow, which is locally aiding rather than opposing the propagation. This enables heat transfer by upstream convection, which is stronger and more far reaching than conduction. In addition, the flame is less prone to extinction by finite rate kinetics effects, which occurs only when the Damköhler number based on the diffusion time in the recirculation region, rather than on the shorter diffusion time in the Navier-Stokes region, becomes of order unity.

\section{Acknowledgments}

This work was partially supported by the DGICYT under contracts PB94-0400 and PB950008, and by the Spanish INTA under contract 4070-0036/1996.

Tarifa C S and Torralbo A M 1967 Flame propagation along the interface between a gas and a reacting medium Proc. 11th Int. Symp. on Combustion pp 533-44

deRis J N 1969 Spread of a laminar diffusion flame Proc. 12th Int. Symp. on Combustion pp 241-52

Williams F A 1976 Mechanisms of flame spread Proc. J6th Int. Symp. on Combustion pp 1281-94

Fernández-Pello A C and Hirano S T 1983 Controlling mechanisms of flame spread Combust. Sci. Technol. 32 1-31

Fernández-Pello A C 1992 Flame spread modeling Combust. Sci. Technol. 39 119-34

Wichman I S 1992 Theory of opposed-flow flame spread Prog. Energy Combust. Sci. 18 553-93

Tarifa C S, Liñán A, Salvá J J, Juste G L, Tizón J M and Cura J M 1990 Study on Combustion Processes in Reduced Gravity Final report LPTR 9004 for ESA Contract 8272/89/F/BZ(SC)

Altenkirch R A, Eichhorn R and Shang P C 1980 Buoyancy effects on flames spreading down thermally thin fuels Combust. Flame 37 71-83

Fernández-Pello A C, Ray R S and Glassman I 1981 Flame spread in an opposed flow: the effect of ambient oxygen concentration Proc. 18th Int. Symp. on Combustion pp 579-87

Hirano T and Kanno Y 1973 Aerodynamics and thermal structures of the laminar boundary layer over a flat plate with a diffusion flame Proc. 14th Int. Symp. on Combustion pp 391-8

Mao C P, Kodama H and Fernández-Pello A C 1984 Convective structure of a diffusion flame over a flat combustible surface Combust. Flame 57 209-36

Hirano T, Noreikis S and Waterman T 1974 Measured velocity and temperature profiles near flames spreading over a thin combustible solid Combust. Flame 23 83-96

Fernández-Pello A C and Williams F A 1976 Experimental techniques in the study of laminar flame spread over solid combustibles Combust. Sci. Technol. 14 155-67

Smith F T 1982 On the high Reynolds number theory of laminar fiows IMA J. Appl. Math. 28 207-8I 
Goldstein S 1930 Concerning some solutions of the boundary layer equations in hydrodynamics Proc. Camb. Phil. Soc. 26 1-30

Hakkinen R J and Rott N 1965 Similarity solutions for merging shear flows II AIAA J. 3 1553-4

Fernández-Pello A C and Williams F A 1977 A theory of flame spread over flat surfaces of solid combustibles Combust. Flame 28 251-277

Higuera F J 1997 Boundary layer separation due to gas thermal expansion Phys. Fluids submitted

Carslaw H S and Jaeger J C 1959 Conduction of Heat in Solids 2nd edn (Oxford: Oxford University Press)

Smith F T 1983 Interacting flow theory and trailing edge separation-no stall J. Fluid Mech. $131219-49$ 